\title{
Physiological Studies of Ornamental Bulb Dormancy
}

\author{
Soudamini Karjee* and Sourav Mahapatra
}

Indian Institute of Horticultural Research, Bengaluru-560089, India

*Corresponding author

\begin{abstract}
A B S T R A C T
Keywords

Bulb dormancy,

Environmental

influence,

Hormonal

regulation,

Physiological

changes and

Sprouting

Article Info

Accepted:

17 March 2019

Available Online:

10 April 2019

Dormancy in bulbs describes a complex phenomenon involving temporal cessation of growth in metabolically active plant parts until the conditions become favourable. The entire physiology of metabolic arrest with its induction and termination is under hormonal, molecular and environmental control. At present, the molecular regulation of dormancy is still remains unclear and least understood. Indian floriculture industry totally depends on import of flower bulbs every year due to the development of deep dormancy in bulbs. Dormant bulb requires an ample of cold treatment period to release its dormancy. Moreover, dormancy limits the production immediately after harvest and importing of bulbs from the other countries increases the chances of entry of exotic pests and diseases which challenges our farmer's economy. To overcome this problem, necessary treatments are required to practice for easy sprouting by modulating the biosynthetic pathway of inhibitory substances.
\end{abstract}

\section{Introduction}

Dormancy is a complex and dynamic morphological, physiological, and biochemical mechanism in which there are no visible external changes or growth and there is a temporary suspension of apparent growth of any plant part having a meristem (Lang et al., 1987).Studies pertaining to bulb dormancy have been conducted by several authors (Wareing and Saunders, 1971; Kamerbeek et al., 1972; Rudnicki, 1974). Dormancy is used to describe the natural phenomenon of growth cessation marked by partial metabolic arrest with its induction and termination under hormonal control. Dormancy has also been reflected to represent a period of intra bulb development (Kamenetsky, 1994). The phenomenon of dormancy appears to be elusive as the process itself; besides it is difficult to describe dormancy as an active or passive period in life cycle. How a metabolically active plant suspends its activities and resumes growth after the conditions become favourable point to the possible existence of a dormancy clock. The physical environment exerts a marked influence on dormancy which is usually 
broken by a period of cold treatment depending on plant species. Altered environmental conditions during the dormancy period have been suggested to trigger the developmental processes leading to ultimate dormancy release (Bewley, 1997). Dormancy can be regarded to be one of the most important factors that has made bulbous plants capable of growing in varied range of climates (Rudnicki, 1974). Bulbs are capable of timing themselves for dormancy. Spring bulbs become dormant in summer and summer bulbs become dormant in winter. Understanding the bulb dormancy therefore seems to be a prerequisite for developing efficient propagation methods as dormancy directly affects storage capacity of bulbs. Predetermined rate of sprout emergence in post dormancy is supposed to be one of the major determinants of storage capacity; besides understanding the mechanisms involved in the regulation of dormancy are important as the dormant geophytes are more resistant to environmental stresses (Borochov et al., 1997). Dormancy has a direct effect on regulation of germination, growth and reproductive development of the plant and often the temporal extent of dormancy has been described by 'dormancy depth' (Kamerbeek et al., 1972). 'Correlative inhibition', 'rest' and 'quiescence' as three phases of dormancy and same have been referred to as 'Ecodormancy', 'Endodormancy' and 'Paradormancy' by Lang et al., (1987). These concepts have been applied to the seeds and buds in general and to geophytes up to some extent. Depending on 'dormancy depths' three types of dormancy have been identified in different sbulbous species viz. lily type dormancy, tulip type dormancy and bulb types without true physiological dormancy (Kamerbeek et al., 1972). In the "lily type" dormancy the bulbs go through a longer depth during which the differentiation of new organs or elongation is completely arrested. The dormancy release takes place slowly spanning over several months and low temperature treatment is a prerequisite for its completion. This dormancy is similar to seed dormancy observed in plants form temperate climates and has been recorded in bulbs like lilies, onions and gladioli. "Tulip type" dormancy is induced soon after flowering and prevents stem elongation. It is characteristic of tulips, daffodils and hyacinths. The third type of dormancy found in plants such as irises is largely driven by environmental factors such as temperature and humidity rather than true physiological requirements and the growth of plant resumes upon the return of favourable conditions. It can be argued that dormancy is an inbuilt and environmentally sustained physiological process; besides the regulation of dormancy can be related to the varied effects of hormones, temperature and light.

\section{Causes of dormancy}

\section{Hormones and dormancy}

In general, it is difficult to decide whether or not a particular substance controls a given physiological process. As Nitch (1957) pointed out, involvement of hormonal factors in the regulation of dormancy should be based on following criteria: First, there must be an indication that a transmissible factor, possibly hormonal in nature, is involved in the induction and release of dormancy. Second, treatment with exogenous growth regulators should impose or break dormancy in various plant organs. Finally, variation in the level of some endogenous growth regulators should parallel the onset and release of dormancy. However, despite many observations fulfilling those criteria, there are also many to the contrary. Below, existing works related to hormones and dormancy will be presented and discussed. The onset and release of dormancy is regulated by the levels of growth inhibitors and promoters which in turn control 
growth and differentiation (Rudniki, 1974). Dormancy induction or release is a collaborative process involving several plant hormones. Exogenous plant growth regulators have also become available for commercial use on flower bulbs; besides a range of physiological processes influenced by PGRs include the control of flowering in Dutch Irises; control of leaf yellowing in lilies; control of marketable plant heights of daffodils, tulips and lilies; and propagation by tissue culture or stem cuttings.

\section{Abscisic acid}

(ABA) has been suspected for a long time to be the inhibitor preventing growth and development of dormant plant organs. In general, ABA content is high in dormant buds and decreases at dormancy release preceding bud break. Treatment with exogenous ABA induced bud dormancy and prevented its release in apples (Guak and Fuchigami, 2001) and induced dormancy in epiphyllous buds of Kalanchoe tubiflora (Palmer and Jasrai, 1996). Endogenous ABA levels are also correlated with seed, tuber and bulb dormancy (Nowak et al., 1975). ABA level in bulbs of dormant type and non-dormant type cultivars of Allium wakegi was similar during the entire time of their development (Yamazaki et al., 1999), which suggests that bulb dormancy is controlled by a different mechanism. In many instances seed dormancy could be released by treatment with fluridone-the inhibitor of carotenoid synthesis that caused a decrease in endogenous ABA levels (Yoshioka et al., 1998).

\section{Auxins}

These have been found to increase during sprouting of bulbs of Polianthese tuberose (Nagar, 1995). IAA-like activity was detected in tulip bulbs during sprouting suggesting the role of auxins in dormancy. The increase in gibberellin and auxin activity has also been recorded during sprouting of stored onion bulbs; the gibberellin -auxin activity was noticed mainly in early sprouts (Thomas, 1969).

\section{Gibberellin}

These like substances have also been identified in bulbs of Allium cepa, Hyacinthus orientalis, Wedgwood Iris, Lilium longiflorum, Narcissus tazetta, Tulipages neriana and Lilium speciosum. In-vitro studies on Lilium speciosum have revealed that addition of Paclobutrazole an inhibitor of GA synthesis reduced dormancy levels in bulblets, suggesting that GA levels are related to dormancy. In many bulbous plants quantitative changes in gibberellins have been reported during bulb development in tulips, irises, daffodils and hyacinths (Nowak, 1976). Possible relation between dormancy and GA levels is still obscure. Whether GA functions through the synthesis of hydrolytic enzyme during dormancy release or increases the thermal sensitivity of bulbs to changed environmental conditions require a rigorous investigation.

\section{Cytokinins and ethylene}

These have been reported to break dormancy in corms of plants such as Gladiolus and Freesia (Tsukamoto, 1972). Ethylene has been found to be effective in breaking dormancy of Fressia corms (Imanishi, 1996). Smoke treatment for dormancy release by eliciting exogenous ethylene production has been found to be effective in Freesia corms (Uyemura and Imanishi, 1983).

\section{Jasmonates and polyamines}

These are plant growth regulators present in all plant tissues analyzed. Polyamines are frequently linked to dormancy. High 
putrescine and low spermidine and spermine levels were associated with the imposition of dormancy in tea, whereas high spermidine and spermine levels were related to dormancy release (Kakkar 1997). Dormancy break of in vitro-cultured spindle tree embryos was followed by an increase in putrescine and spermidine concentrations and in the activities of their biosynthetic enzymes, especially arginine decarboxylase. Dormancy break was also correlated with a decrease of tyramine (Beranger-Novat et al., 1997).

\section{Environment and dormancy}

Temperature along with light and hormones appears to regulate the dormancy cycle; besides temperature rather than photoperiod has been suggested to play a primary role in the regulation of bulb dormancy.

\section{Phytochrome}

Although phytochrome is not a plant hormone, it acts as a light receptor, and therefore plays an important role in the induction and release of dormancy. It has been demonstrated that the light stimulus is perceived by phytochrome present in leaves, but the effect is expressed in terminal or axilary buds. It follows the cessation of growth and the formation of resting buds must be mediated by a transmissible element, presumably of hormonal nature, which is synthesized in leaves under short day conditions and transported to the shoot apex. Some studies demonstrate that, the existence of a growth inhibitor present in leaves exposed to short day conditions (Tumanov et al., 1974). Alleweldt and During (1972) reported that this transmissible growth inhibitor may be abscisic acid, but this finding was never confirmed by other authors. There are also reports that cytokinins and/or gibberellins, together with the phytochrome system, may participate in photoperiodic signaling. However, there is a poor understanding of the mechanism(s) whereby these signals are integrated at the molecular level. Current models propose that light and phytohormones might act independently or sequentially through common signal transduction intermediates to control the same downstream responses (Thomas et al., 1997).

\section{Temperature}

Various physiological aspects of low and high temperature treatments have been studied extensively in the major geophytes with the aim of standardizing commercial bulb storage and production; however the mechanism of dormancy release with temperature manipulation is still unclear. The temperature and period required for the release from dormancy differs between various species and genotypes. Temperature treatments have been extensively used to alter the dormancy or vegetative growth periods to obtain desired flowering of bulbous plants. Varying low degrees of temperatures have been developed as successful protocols for bulb storage. High temperatures have been found to play a role in the release of dormancy in Iris bulbs (Tsukamoto and Ando, 1973). By placing Iris bulbs at a high temperature after lifting, leaf production was found to continue without flower formation; but if high temperature treatment was followed by reduced temperature flowering was induced. These findings suggest that high temperatures reduce dormancy promoters thereby enabling flowering.

\section{Biochemical causes of dormancy}

\section{ABA biosynthesis}

It plays a major role in development of dormancy. It has two phases described below that how the abscisic acid formation takes place and the complete mechanism behind this. 


\section{Early, steps in ABA biosynthesis}

Plant isoprenoids are derived from either a cytoplasmic acetate/ mevalonate pathway shared with animals and fungi or a plastidic MEP pathway, with first committed molecule 2C-methyl-D-erythritol-4-phosphate (RuizSola and Rodriguez-Concepcion, 2012). The next major phase of ABA biosynthesis is production of carotenoids. Sequential condensation reactions catalyzed by geranylgeranyl diphosphate synthase (GGPPS: AT4G36810) add one isoprene unit at a time to successively generate $\mathrm{C} 10, \mathrm{C} 15$ and C20 molecules (geranyl diphosphate, farnesyl diphosphate, and geranylgeranyl diphosphate (GGPP), respectively). Subsequent head to head condensation of two GGPPs by phytoene synthase (AT5G17230) produces the $\mathrm{C} 40$ skeleton that will become phytoene, the first committed carotenoid. Phytoene is subjected to four consecutive desaturation (dehydrogenation) reactions that lead to the formation of lycopene. These reactions are catalyzed by two homologous enzymes: phytoenedesaturase (AT4G14210) and $\alpha$-carotene desaturase (AT3G04870). Carotenoid desaturation in plants requires a third enzyme, carotenoid isomerase (CRTISO: AT1G06820), but photoisomerization can supply this function in tissues with adequate light penetration. Only $\beta$-carotene is further metabolized to ABA via zeaxanthin; the $\alpha$-carotene branch leads to lutein synthesis. However, $\beta$-carotene pool sizes in photosynthetic tissues are tightly regulated such that only a small proportion is metabolized to zeaxanthin. Production of zeaxanthin, the first oxygenated carotenoid, is catalyzed by $\beta$-carotene hydroxylases encoded by two homologous genes (BCH1 and BCH2: AT4G25700 and AT5G52570) in Arabidopsis and many other species. ZEP is a chloroplast-imported protein of bacterial origin. The reactions catalyzed by ZEP can be reversed by violaxanthin de-epoxidase (VDE:
AT1G08550) to produce more photoprotective zeaxanthin in response to a sudden increase in light intensity; this process is known as the xanthophyll cycle.

\section{Late, specific steps in ABA biosynthesis}

The final plastid-localized steps in $\mathrm{ABA}$ synthesis are conversion to another C40 compound, trans-neoxanthin, isomerization of either (trans)-violaxanthin and transneoxanthin to their 9-cisisomers, and cleavage by 9-cis-epoxycarotenoid dioxygenase (NCED) to release the $15^{\circ} \mathrm{C}$ compound xanthoxin. Neoxanthin synthesis was recently found to depend on the product of the ABA4 locus (AT1G67080), a highly conserved unique plastid membrane-localized protein (North et al., 2007). ABA4 is expressed constitutively and the basal expression levels appear sufficient for ABA synthesis under stress conditions, indicating that transcript levels are not rate-limiting. According to the previous suggestionthat NCED could use either cis-neoxanthin or cis-violaxanthin as substrates. Xanthophyll cleavage by NCED is the first committed step in ABA biosynthesis, and is rate-limiting (Nambara and MarionPoll, 2005). Consequently, NCED expression is tightly regulated in response to stress or developmental signals, as well as diurnally. NCEDs are encoded by multigene families in all species analyzed, with differential expression. Abscisic Acid 5 of 36 Xanthoxin is converted to $\mathrm{ABA}$ by a series of oxidative steps via the intermediate abscisic aldehyde. Several additional loci contribute to these last two steps. ABA2 (AT1G52340) encodes a short chain dehydrogenase/reductase-like (SDR1) enzyme catalyzing production of abscisic aldehyde. The final step creating the carboxyl group at the end of the side chain is catalyzed by abscisic aldehyde oxidase (AAO). As described above, ABA is primarily synthesized in vascular tissues and transported to target tissues. This transport 
occurs in both xylem and phloem, permitting transport in both directions between roots and

shoots leading to the development of dormancy.

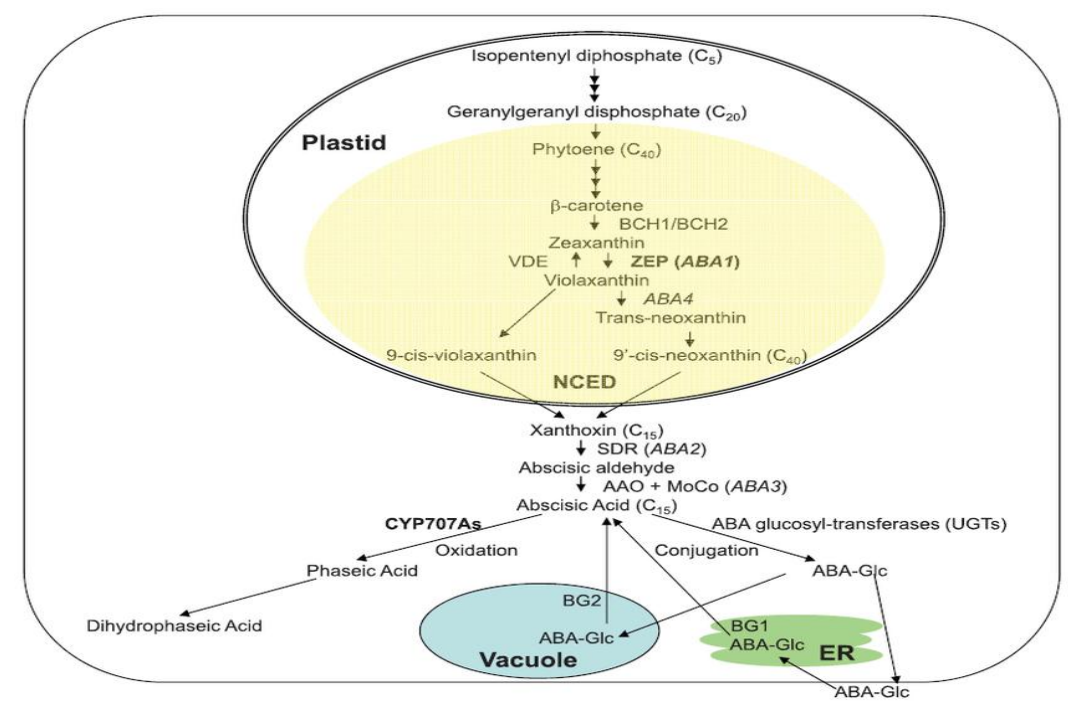

ABA metabolic pathways(Finkelstein,2002)

\section{Starch metabolism}

In the non-photosynthetic cells of higher plants generally, sucrose which is transported from the photosynthetic apparatus, is cleaved to its constituent monosaccharides, hexoses or phosphorylated hexoses, which can then be used either in metabolic or biosynthetic reactions. Sucrose is degraded by four different enzymatic mechanisms. Firstly, it is hydrolysed into hexoses (glucose and fructose) by cell wall invertase in the apoplast. Hexoses generated are then transported into the cytosol by hexose transporters. Secondly, cytosolic sucrose transported from the phloem by sucrose transporters may also be taken up into vacuoles for hydrolysis by vacuolar invertase (VIN). Both the remaining two mechanisms take place in the cytosol. Thirdly, sucrose is hydrolysed into hexoses by cytoplasmic invertase (CIN). Hexoses are converted into hexose-6-phosphates by hexokinase. Fructose-6-phosphate (F-6-P) is converted into glucose-6 phosphate (G-6-P) by glucose phosphate isomerase, but on the other hand, F-6-P can synthesis sucrose via sucrose phosphate synthase. Fourthly, sucrose is reversibly converted into fructose and uridine diphosphate glucose (UDPG) by sucrose synthase. Then UDPG is further metabolized to glucose-1-phosphate (G-1-P) by the action of UDPG pyrophosphorylase. G-1-P, which can also be transformed from G-6-P by phosphoglucomutase, serves as a precursor of adenosine diphosphate glucose (ADPG) by ADPG pyrophosphorylase. Both G-1-P and G-6-P are translocated into the amyloplasts via phosphate translators, whereas ADPG is translocated via ADPG transporters. Then, starch biosynthesis occurs in the amyloplast leading to the development of dormancy. The detailed process is described below.

\section{Dormancy release}

Dormancy release initiates a metabolism upsurge with the constant input of sugars for maintaining the processes of growth and development. Dormancy release in various bulbous crops has long been associated with abscisic acid (Nagar, 1995; Yamazaki et al., 1999). 


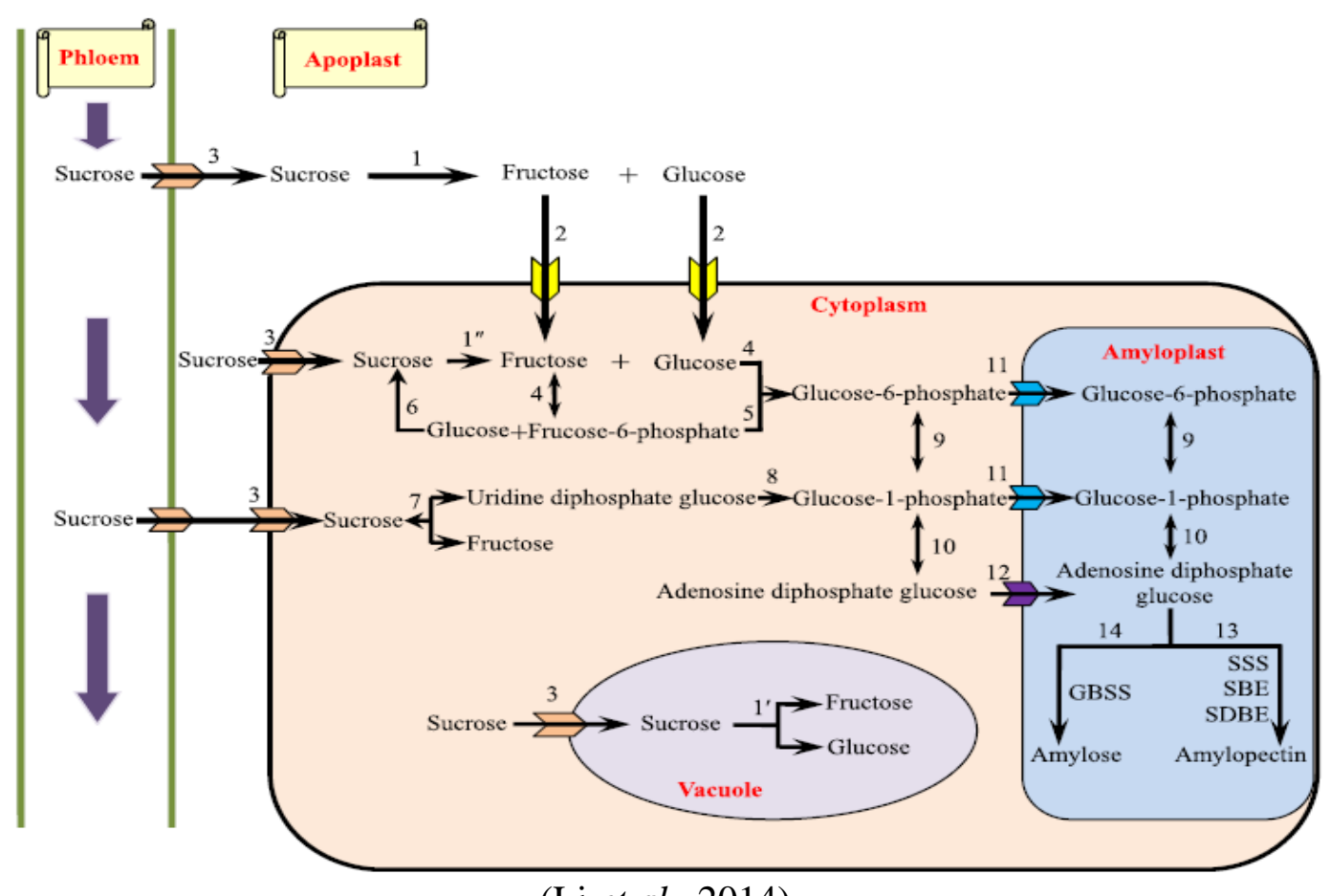

(Li et al., 2014)

Endogenous levels of ABA have been attributed to play a major role in dormancy development in lily bulbs (Kim et al., 1993). The decrease in the endogenous ABA level during bulb storage of Lilium rubellum has been correlated with dormancy-release. Dormant bulbs of Iris have been reported to contain high levels of ABA, which declines at the release of dormancy (Okubo, 1992). ABA has been found to be involved in induction and maintenance of dormancy in bulbs of Polianthes tuberose (Nagar, 1995). It appears that dormancy involves synchronous participation of endogenous hormones along with temperature and light however regulation of endogenous plant growth regulators at genetic level is still a matter of investigation in bulbous plants.

\section{Signs of dormancy release}

Termination of dormancyhas been marked by an increase in the activity of various hydrolytic enzymes and breakdown of stored reserves in bulb tissue (Nowak et al., 1974). It has been seen that $\alpha$-amylase activity and sucrose content increased during the cold storage period in hyacinth shoots (Sato et al., 2006). Storage of iris bulbs at $10-13^{\circ} \mathrm{C}$ not only stimulated development of new buds and flower initiation but also an increased starch hydrolysis, respiration and peroxidase activity (Halevy et al., 1964). It appears from the studies that bulbs require a minimum critical mass before dormancy release ensuring storage of enough reserve material for development. Maintenance of low oxygen tension has also been found to be effective in breaking bulb dormancy in Lilium and this method has been preferred to conventional hot water soaking of vernalised bulbs (Wakakitsu, 2004). The flavonoids present as glycosides have been ascribed to play an important role in the release of dormancy. Differential levels of endogenous polyamines in tuberose (Polianthes tuberosa) have been suggested to alter dormancy. Maintenance of high free putrescine, besides low spermine and spermidine levels have been associated with initial stages of dormancy whereas high spermine and spermidine levels have been shown to be associated with the release of 
dormancy. Water status has been shown to register an increase during dormancy release and the storage polysaccharides are cleaved to low-molecular weight sugar molecules (Kamenetsky, 2002).

\section{Significance of dormancy release}

It can ensure the production of cut flower immediately after the harvest of bulbs. If it is possible there is no need to go for cold storage period to break the dormancy. Although it can save the costs of cold storage if chemical method of breaking dormancy is successful. Moreover, dormancy release will reduce the duration of crop cycle i.e. 24 weeks of dormancy period can be reduced out of 48 weeks from the total bulb cycle resulting in doubling the farmer's income. It allows the farmer to save his own bulbs and avoids in purchasing the bulbs every time from the company. Also the entry of new pests and diseases through importing bulbs from outside country can be minimised.

In conclusion, as we confirmed dormancy is an important and complex physiological process that is controlled by both developmental and environmental factors. The genetic and molecular mechanisms that govern bulblet development and dormancy still remain unexplored and unclear. ABA is the main component which plays an important role in dormancy induction through biosynthesis of AtNCED (9-cis epoxycarotenoid dioxygenase) (NCED6 and NCED9) in bulbous crops. We found cold treatment at $4^{\circ} \mathrm{C}$ for $6-8$ weeks is an effective method to break dormancy of a large number of ornamental bulbous and tuberous plants. Gibberellins (GA), Ethylene, Brassinosteroids (BR), Cytokinins (BA) and N-containing compounds releases dormancy and counteracts inhibitory ABA effects. Phytochromes modulate endogenous levels of GA and ABA.

\section{Future prospects}

There are still more plant biology avenues that needs to be investigated, includes histological changes, osmolytes accumulation, water and nutritional relations and functional genomics under normal or stressful conditions. At present, there is no consistent theory which would explain the mechanism of hormonal control of dormancy. New approaches, especially in molecular biology, should provide new information in this important field. Expression of dormancy genes can be studied to silence them so that the problem of dormancy can be overcome. The pathway of ABA signal transduction leading to regulation of various physiological processes, is very complex and not yet elucidated which should be well understood for further research work. Breaking of dormancy in-vitro using bioreactor with chemically/Temperature will scales-up the production system and will provide the advantages of programmable production, cost reduction, and easy control over dormancy.

\section{References}

Alleweldt, G. and H. During. 1972. Einfluss der Photoperiode auf Wachstum und Abscisinsauregehalt der Rebe. Vitis. 11: 280-288.

Beranger-Novat, N., J. Monin. and J. MartinTanguy. 1997. Polyamines and their biosynthetic enzymes in dormant embryos of the spindle tree (Euonymus europaeus L.) and in dormancy break obtained after treatment with gibberellic acid. Plant Growth Regulat. 21: 65-70.

Bewley, J.D., 1997. Seed dormancy and germination. Plant Cell. 9: 1055-1066.

Borochov, A., Spiegelstein, H. and Weiss, D. 1997. Dormancy and storage of geophytes. Acta Horticulturae, 430: 405-409.

Finkelstein, R., Reeves, W., Ariizumi, T. and 
Steber, C. 2008. Molecular aspects of seed dormancy. Annu. Rev. Plant Biol. 59: 387-415.

Finkelstein, R.R., Gampala, S.S.L. and Rock, C.D. 2002. Abscisic acid signaling in seeds and seedlings. Plant Cell. S15S45.

Guak, S. and L.H. Fuchigami. 2001. Effects of applied ABA on growth cessation, bud dormancy, cold acclimation, leaf senescence and $\mathrm{N}$ mobilization in apple nursery plants. J. Hort. Sci. Biotech. 76: 459-464.

Halevy, A. H. andShoub, J. 1964. The effect of cold storage and treatment with gibberellic acid on flowering and bulb yields of Dutch iris. Journal of Horticultural Science. 39(2): 120-129.

Imanishi, H. 1996. Ethylene as a promoter for flower induction and dormancy breaking in some flower bulbs. In VII International Symposium on Flowerbulbs430: 79-88.

Kakkar, R.K. and P.K. Nagar. 1997. Distribution and changes in endogenous polyamines during winter dormancy in tea (Camellia sinensisL. (O) Kuntze). J. Plant Physiol. 151: 63-67.

Kamenetsky, R. 1994. Life cycle, flower initiation, and propagation of the desert geophyte Allium rothii. International Journal of Plant Sciences, 155(5): 597605.

Kamerbeek, G. A., Beijersbergen, J. C. M. and Schenk, P. K. 1972. Dormancy in bulbs and corms. In Proceeding of XVIIIth Intl. Horticultural Congress, Tel Aviv. 5: 233-239.

Kim, K. S., Davelaar, E. and De Klerk, G. J. 1994. Abscisic acid controls dormancy development and bulb formation in lily plantlets regenerated in vitro. Physiologia Plantarum, 90(1): 59-64.

Kumar, P. and Raju, D. 2007. Dormancy in Gladiolus: the cause and remedy - a review. Agric. Rev. 28 (4): 309-312.
Lang G., Early J., Martin G. and Darnell R. 1987. Endo-, para- and ecodormancy: physiological terminology and classification for dormancy research. Hort Science. 22: 371-377.

Li, X.Y., Wang, C.X., Cheng, J.Y., Zhang, J., Teixeira da Silva, J. A., Liu, X.Y., Duan, X., Li, T.L. and Sun, H.M. 2014. Transcriptome analysis of carbohydrate metabolism during bulblet formation and development in Lilium davidii var. unicolor. BMC Plant Biology 14:358.

Nagar, P.K. 1995. Changes in abscisic acid, phenols and indoleacetic acid in bulbs of tuberose (Polianthes tuberosa L.) during dormancy and sprouting. Sci. Horti. 63: 77-82.

Nambara, E. and Marion-Poll, A. 2005. Abscisic acid biosynthesis and catabolism. Ann. Rev. Plant Biol. 56: 165-185.

Nitsch, J.P. 1957. Growth responses of woody plants to photoperiodic stimuli. Proc. Am. Soc. Hort. Sci. 70: 512-525.

North, H.M., Almeida, A.D., Boutin, J-P., Frey, A., To, A., Botran, L., Sotta, B. and Marion-Poll, A. 2007. The Arabidopsis ABA-deficient mutant $a b a 4$ demonstrates that the major route for stress-induced ABA accumulation is via neoxanthin isomers. Plant J. 50: 810-824.

Nowak, J., J. Ross, R.M. Rudnicki. and M. Saniewski. 1975. The presence of abscisic acid in Hyacinthus orientalis bulb. Phytochemistry. 12: 5015-5016.

Okubo, H. 1992. Dormancy in bulbous plants. In VI International Symposium on Flower Bulbs. 325: 35-42.

Palmer, J.P. and Y.T. Jasrai. 1996. Precocious growth and effect of ABA: Encapsulated buds of Kalanchoetubiflora. J. Plant Bioch. Biotech. 5: 103-104.

Rudnicki, R. M. and Nowak, J. 1976. Studies on the physiology of hyacinth bulbs 
(Hyacinthus orientalis L.) VI. Hormonal activities in hyacinth bulbs during flower formation and dormancy release. Journal of Experimental Botany, 27(2): 303-313.

Rudnicki, R. M. 1969. Studies on abscisic acid in apple seeds. Planta. 86: 65-85.

Ruiz-Sola, M. A. and Rodriguez-Concepcion, M. 2012. Carotenoid biosynthesis in Arabidopsis: A colorful pathway. The Arabidopsis Book.10: e0158.

Sato, A., Okubo, H. and Saitou, K. 2006. Increase in the expression of an alphaamylase gene and sugar accumulation induced during cold period reflects shoot elongation in hyacinth bulbs. Journal of the American Society for Horticultural Science. 131(2): 185-191.

Thomas, T.H., P.D. Hare. and J. Van Staden. 1997. Phytochrome and cytokinin responses. Plant Growth Regulat. 23: 105-122.

Tsukamoto, Y. 1972. Breaking dormancy in the gladiolus corm with cytokinins. Proceedings of the Japan Academy, 48(1): 34-38.

Tumanov, I.I., G.V. Kuzina. and L.D. Karnikova. 1974. Increasing the capacity of woody plants for frost hardening by treatment with extracts of naturally occurring growth inhibitors. FiziologiyaRastenii.21: 380-390.

Uyemura, S. and Imanishi, H. 1983. Effects of gaseous compounds in smoke on dormancy release in freesia corms. Scientia Horticulturae, 20(1): 91-99.

WakakitsuAmaki, W. A. andWakanoriAmaki, W. A. 2004. Dormancy Release of Easter Lily Bulb by Low O2 Concentration Treatments. In $I X$ International Symposium on Flower Bulbs. 673: 591-594.

Wareing, P. F. and Saunders, P. F. 1971. Hormones and dormancy. Annual Review of Plant Physiology, 22(1): 261288.

Yamazaki, H., Nishijima, T., Yamato, Y., Hamano, M., Koshioka, M. and Miura. H. 1999. Involvement of abscisic acid in bulb dormancy of Allium wakegi Araki. II. A comparison between dormant and non dormant cultivars. Plant Growth Regulat. 29: 195-200.

Yoshioka, T., T. Endo. and S. Satoh. 1998. Restoration of seed germination at supraoptimal temperatures by fluridone, an inhibitor of abscisic acid biosynthesis. Plant Cell Physiol. 39: 307-312.

\section{How to cite this article:}

Soudamini Karjee and Sourav Mahapatra. 2019. Physiological Studies of Ornamental Bulb Dormancy. Int.J.Curr.Microbiol.App.Sci. 8(04): 2305-2314.

doi: https://doi.org/10.20546/ijcmas.2019.804.269 